\title{
La evaluación del presupuesto por resultados y su impacto en la seguridad ciudadana del Perú
}

\author{
Aldo Fernando Rejas de la Peña \\ aldobakul@gmail.com, arejas@ucvvirtual.edu.pe \\ ORCID: 0000-0002-8594-8620 \\ Lic. En Educación Secundaria Formación Laboral \\ con mención en Administración y Contable \\ y Lic. En Administración y Ciencias Policiales, \\ Mg. Psicología Educativa Doctorando en Gestión Pública y Gobernabilidad, \\ Universidad Cesar Vallejo. \\ Eddy Ronald Diaz Salvatierra \\ Eddy.diaz@upn.edu.pe, ediazsa4@ucvvirtual.edu.pe \\ ORCID: 0000-0001-6164-6460 \\ Lic. En Trabajo Social y Lic. en Educación, \\ especialidad Historia y Geografía, \\ Mg. con mención en docencia Universitaria, \\ Dr. En Políticas Públicas y Gobernabilidad, \\ Universidad César Vallejo, Universidad Privada del Norte. \\ Herbert Troya Acha \\ hebertroya@gmail.com \\ ORCID: 0000-0003-0938-0380 \\ Lic. En Administración en ciencias policiales \\ Escuela de oficiales de la PNP \\ Universidad Cesar Vallejo \\ Lima - Perú
}

\section{RESUMEN}

El objetivo del presente es articular los esfuerzos de todas las instituciones involucradas para contrarrestar que demanda la implantación de la Estrategia Nacional de Seguridad Ciudadana 2018-2023; teniendo su implicancia en la asignación de recursos públicos necesarios para la atención de las demandas ciudadanas; sobre los resultados obtenidos de los presupuestos asignados, identificar aspectos positivos o negativos de la gestión de los presupuestos públicos para mejorar las políticas públicas. La investigación es de un enfoque cualitativo, estudio de caso, de muestra no probabilística, eligiéndose base el presupuesto de la Municipalidad Distrital de Ate. En los resultados obtenidos se identifica 
que los presupuestos se han incrementado en el tiempo, pero no así sus resultados. Concluyendo que la evaluación de la ejecución presupuestal de la Municipalidad distrital de Ate, se advierte que pese a que ha existido un incremento sostenido en la ejecución de recursos públicos a favor de la seguridad ciudadana en el programa presupuestal 030; La disponibilidad presupuestal que tiene cada entidad municipal de acuerdo a su recaudación de los arbitrios serenazgo o seguridad ciudadana y las trasferencias que realizan los gobiernos regionales o gobierno central el cual es muy asimétrico identificándose asignaciones presupuestales por habitante y por año.

Palabras clave: bienestar social, gestión por resultados, presupuesto por resultados, seguridad ciudadana y seguridad social. 


\title{
The evaluation of performance budgeting and its impact on citizen security in Peru
}

\begin{abstract}
The objective of this document is to articulate the efforts of all the institutions involved to counteract the demands of the implementation of the National Strategy for Citizen Security 2018-2023; having its implication in the allocation of public resources necessary for the attention of citizen demands; on the results obtained from the assigned budgets, identify positive or negative aspects of the management of public budgets to improve public policies. The research is of a qualitative approach, case study, non-probabilistic sample, choosing the budget of the District Municipality of Ate as the base. In the results obtained, it is identified that the budgets have increased over time, but not their results. Concluding that the evaluation of the budget execution of the District Municipality of Ate, it is noted that despite the fact that there has been a sustained increase in the execution of public resources in favor of citizen security in the budget program 030; The budget availability that each municipal entity has according to its collection of serenazgo or citizen security excise taxes and the transfers made by the regional governments or the central government, which is very asymmetric, identifying budget allocations per inhabitant and per year.
\end{abstract}

Key words: social welfare, management for results, budget for results, citizen security and social security.

Artículo recibido: 02 enero 2022 Aceptado para publicación: 28 enero 2022 Correspondencia: aldobakul@gmail.com Conflictos de Interés: Ninguna que declarar 


\section{INTRODUCCIÓN}

Entre enero 2013 y setiembre de 2018 la delincuencia se mantuvo como uno de los principales problemas del país percibidos por la ciudadanía, aunque a partir de 2017 pasó del primer al segundo lugar después de la corrupción. En 2013, el 40.4\% de la población consideraba a la delincuencia como el principal problema del país seguido de la pobreza con $38.9 \%$, y en setiembre de 2018 dicho porcentaje pasó a $39.6 \%$, precedido por la corrupción que pasó a ser el principal problema del país con 60.1\% (Ministerio del interior, 2018 p. 9), lo que demuestra el incremento de la inseguridad ciudadana que demanda la implementación de la Estrategia Nacional de Seguridad Ciudadana 2018 2023 cuyo objetivo radica en articular los esfuerzos de todas las instituciones involucradas para contrarrestar este flagelo; lo que implica la asignación de recursos públicos necesarios.

El Decreto Legislativo $\mathrm{N}^{\circ} 1140$ establece como principio que la gestión presupuestaria tiene que estar orientada al logro de resultados a favor de la población y que se estructura, gestiona y evalua bajo la lógica del Presupuesto por Resultados (PpR), la cual constituye una estrategia de gestión pública que vincula los resultados a productos y resultados medibles a favor de la población a través de instrumentos : Programas presupuestales seguimiento - evaluación e incentivos presupuestarios (ministerio de economía y finanzas, 2018), para contrarrestar la amenaza de la inseguridad ciudadana, el Ministerio de Economía y Finanzas ha implementado el Programa Presupuestal 030: Reducción de Delitos y Faltas que afectan a la Seguridad Ciudadana, mediante el cual destina recursos públicos a los gobiernos central, regional y local, con la finalidad de implementar la política pública de seguridad ciudadana en el ámbito de sus competencias y jurisdicciones (Ministerio de economía y finanzas, 2021).

En la actualidad se encuentra en la agenda pública los presupuestos destinados a Seguridad Ciudadana, para el caso de Bogotá, se teniendo como elemento de justificación que la obtención de resultados favorables es directamente proporcional a los recursos económicos destinados para ello (Becerra, 2018).

En la Municipalidad Distrital de Ate El gasto publico ejecutado en el PpR sobre seguridad ciudadana para el periodo 2012 hasta el 2018, tuvo un constante incremento, en el año 2012 el Presupuesto Institucional Modificado (PIM) que fue de S/. 2895214073.00 soles, el año 2018 está alcanza la cifra de S/. 5739209179.00 soles (Delgadillo Mendoza, 
2018), donde se advierte que dicho presupuesto se ha multiplicado por $250 \%$ perido, sin embargo, los resultados alcanzados no han logrado disminuir los índices de victimización ni la sensación de seguridad ciudadana en el distrito de Ate.

Una de las causas de la inseguridad urbana es la presencia de ciudadanos en situación de desempleo, pobreza, marginación, Lima concentra asentamientos desordenados carentes de servicios básicos" y sobre el problema existe percepción baja de aceptación con respecto al Estado para solucionar este problema.

Sobre el impacto de la delincuencia, falta de seguridad y la corrupción en nuestro país, en el estudio de opinión publica a nivel nacional urbano y rural" realizado por CPI en el año 2017, para el cual esta entidad utilizo como universo de encuesta la población urbana y rural del país (CPI, 2017).

El tema de la inseguridad ciudadana ha sido un problema crítico del gobierno central a través del Ministerio del Interior, los Gobiernos Regionales y principalmente de los Gobiernos locales, en todas las gestiones de cualquier tendencia política, no se ha trabajado con visión de futuro, con la finalidad de articular las estrategias nacionales, regionales y locales y los presupuestos asignados a través del programa presupuestal 030: Reducción de la comisión de delitos y faltas que afectan la Seguridad Ciudadana, que permitan trabajar el problema a largo plazo, sin embargo, hay una política pública denominada Estrategia Nacional de Seguridad Ciudadana 2018 - 2023.

En la jurisdicción de la Municipalidad Distrital de Ate, la asignación del presupuesto para seguridad ciudadana, es del orden de 54 soles por habitante/año, mientras que en la municipalidad de San Isidro es en promedio 500 soles por habitante año, lo que demuestra las diferencias que existen entre jurisdicciones en la misma capital de la república para abordar un problema que es horizontal para todos los pobladores; es ahí donde se tiene el principal problema, que genera exclusión de servicios de seguridad ciudadana de calidad en jurisdicciones provinciales o distritales que tienen escasos recurso para ser destinados a ese rubro en contraste con otros distritos de mayor capacidad de recursos.

En Ate, pese a que se ha multiplicado el presupuesto destinado a Seguridad Ciudadana, los resultados no se han reflejado en esa misma dimensión, incluso existe un incremento de la inseguridad en la jurisdicción, al identificar las causas se tiene que al existir la aplicación de los presupuestos destinados a Seguridad Ciudadana individualmente por cada distrito según su capacidad de percepción de recursos o asignaciones del gobierno 
central, es que existe la migración del delito que traspasa las fronteras del distrito que hace muy difícil medir los resultados obtenidos en la aplicación del presupuesto por resultados.

Demostrar el impacto del Presupuesto por Resultados del Programa presupuestal 030: Reducción de los delitos y faltas que afectan la Seguridad Ciudadana en la reducción de la inseguridad ciudadana en Ate, con la finalidad de revertir los índices de victimización y sensación de inseguridad.

\section{ESTRATEGIAS METODOLÓGICAS}

Tipo de investigación Básica: o "sustantiva" Recibió este nombre simplemente por su interés en los objetivos comerciales, su estimulación se basa en la curiosidad, "La alegría ilimitada de descubrir nuevos conocimientos, y como han dicho otros, el amor de la sabiduría por la sabiduría". Es la base para la investigación técnica o aplicada. (Esteban, 2018).

Para la presente investigación se aplicó un estudio de caso (Hernandez-Sampieri, Mendoza, 2018), al ser esta una investigación de enfoque cualitativo, El término Cualitativo, basa su origen en 2 conceptos. Cualidad: "Fulano tiene una gran cualidad: es sincero". Y Calidad: resalta la integridad y la comprensión "control de calidad", Representa la naturaleza y esencia completa e integral del producto (Salazar, 2020). Se piensa que el método es la continuidad de las polillas y se crean onomatopeyas en la comunicación que implican estos procesos. Investigación cualitativa de alta calidad, incluidas descripciones estratégicas y marcos analíticos (Stenfors, et al 2020). Se caracteriza por un énfasis en la investigación interpretativa, naturalista y holística; compartiendo una hipótesis sobre la existencia de múltiples realidades (Anderson, 2017). Estudio de caso ayuda a comprender mejor los escenarios, la descripción de los hechos que influyen en el comportamiento del problema, porque permite que se pueda elegir con totalidad libertad los conceptos y las teorías” (Ramírez -Sánchez, 2019), Los estudios de casos tratan sobre proporciona excelentes métodos analíticos para situaciones específicas. Preguntas prácticas que ayudan a comprender los fenómenos. El objeto de investigación, siempre parte de la meta a alcanzar nuevas perspectivas y explicaciones (Enrique \& Barrio, 2018). En la investigación se formuló una muestra no probabilística, eligiéndose base el presupuesto de la Municipalidad Distrital de Ate. 
Además, se aplicado el análisis documental (Laban, 2019), constituido por documentos, tanto en formatos físicos como digital; el Análisis documental contribuye a la definición de propósito de la investigación o estudio, estableciéndose un diseño de análisis sistemático bibliográfico y busque de literatura utilizando diversas herramientas que articulas el proceso selectivo de antecedentes (Casasempere-Satorres \& VercherFerándiz, 2020).

Como método de investigación se fundamenta en Hernández-Sampieri y Mendoza (2018) en la ruta cualitativa prevalece el razonamiento lógico o inductivo, que va de lo particular a lo general. Primero, los individuos son descubiertos y descritos para crear teorías. Por ejemplo, en un estudio cualitativo típico, el investigador entrevista a un sujeto, analiza la información obtenida y saca conclusiones; luego, interroga a la otra persona; revisa la nueva información y considera sus hallazgos y conclusiones; da seguimiento a los informes sobre el progreso de la investigación.

\section{RESULTADOS Y DISCUSIÓN}

Los resultados obtenidos respecto a la aplicación del presupuesto por resultados asignados a la Seguridad Ciudadana son:

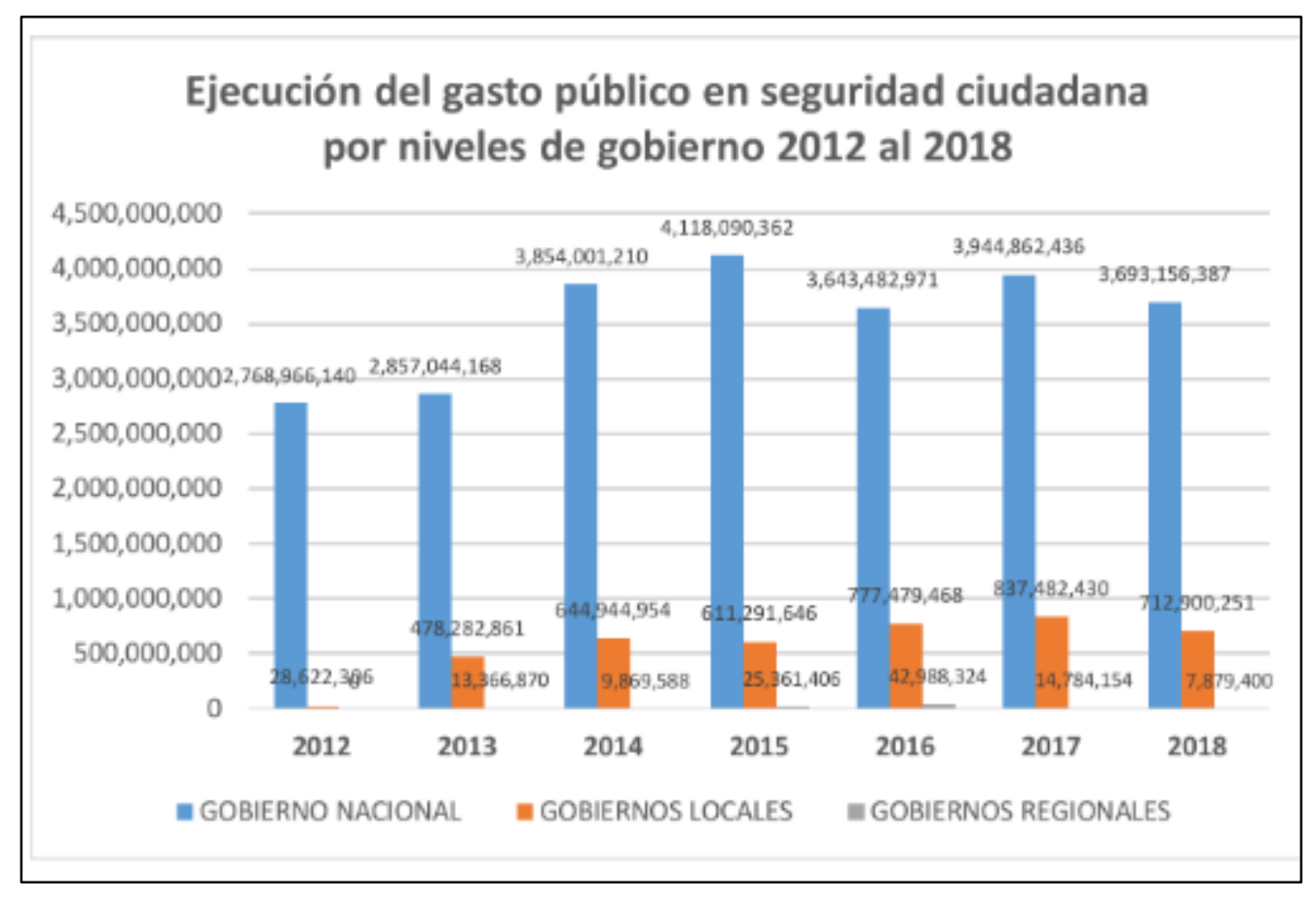

Fuente: SIAF amigable MEF

Conforme se puede apreciar en el gráfico, tenemos que el presupuesto asignado a

Seguridad Ciudadana se encuentra concentrado principalmente en el gobierno central, 
específicamente al Sector Interior - Policía Nacional del Perú y luego se advierte que se encuentran los gobiernos locales.

Sin embargo, a nivel de gobiernos locales este se encuentra atomizado, teniendo en consideración que el Perú cuenta con 195 municipalidades provinciales y 1845 municipalidades distritales y cada una de ellas, de la recaudación de arbitrios por concepto de Seguridad Ciudadana o Serenazgo, perciben los recursos destinados a contrarrestar este flagelo en coordinación con las dependencias policiales que se encuentran en las jurisdicciones de cada municipalidad.

\begin{tabular}{|c|c|c|c|c|c|c|c|}
\hline \multicolumn{8}{|c|}{$\begin{array}{l}\text { Ejecución del gasto público del programa presupuestal 0030: reducción de delitos y faltas que afectan la seguridad ciudadana por } \\
\text { niveles de gabierno }\end{array}$} \\
\hline \multicolumn{8}{|l|}{ (ENSOLS) } \\
\hline $\begin{array}{l}\text { NIVEEES DE } \\
\text { GOBBERNO }\end{array}$ & 2012 & 2013 & 2014 & 2015 & 2016 & 2017 & 2018 \\
\hline $\begin{array}{l}\text { GOBIERNO } \\
\text { NACIONOLL }\end{array}$ & $27689,966,140$ & $2,857,044,163$ & $3,554,001,210$ & $4,118090,352$ & $3.443,462.971$ & $3,944,862,436$ & $3689,156,377$ \\
\hline $\begin{array}{l}\text { GOBERENOS } \\
\text { LOCMILS }\end{array}$ & $22,622,305$ & $478,282,831$ & $644,044,954$ & $611,291,346$ & $777,479,468$ & $897,482,430$ & $712,9002,251$ \\
\hline $\begin{array}{l}\text { GOBBERNOS } \\
\text { REGONALES }\end{array}$ & 0 & $13,366,870$ & $9,869,588$ & $25,361,406$ & $42,983,324$ & $14,784,134$ & $7,879,400$ \\
\hline TOTAL & $2,797,588,446$ & $3,348,693,899$ & $4,508,815,752$ & $4,754,743,414$ & $4,460,950,763$ & $4,797,129,020$ & $4,413,936,038$ \\
\hline \multicolumn{8}{|l|}{ (ENS) } \\
\hline $\begin{array}{l}\text { NINELES DE } \\
\text { GOBIERNO }\end{array}$ & 2012 & 2013 & 2014 & 2015 & 2016 & 2017 & 2018 \\
\hline $\begin{array}{l}\text { GOBIERND } \\
\text { MACIONOLL }\end{array}$ & 99.0 & 85.3 & 85.5 & 83.6 & 81.5 & 822 & 33.7 \\
\hline $\begin{array}{l}\text { GOBlERNDS } \\
\text { LOCMLES }\end{array}$ & 1.0 & 14.3 & 14.3 & 12.9 & 17.4 & 17.5 & 162 \\
\hline $\begin{array}{l}\text { GOBBERNOS } \\
\text { REGONALES }\end{array}$ & 0.0 & 0.4 & 0.2 & 0.5 & 10 & 03 & 02 \\
\hline TOTAL & 100.0 & 100 & 100 & 100 & 100 & 100 & 100 \\
\hline
\end{tabular}

Conforme se puede advertir, el presupuesto asignado a seguridad ciudadana en el Programa Presupuestal. 030. Reducción de delitos y faltas que afectan la seguridad ciudadana, desde el periodo 2012 al 2018, existe un decrecimiento porcentual de parte del Gobierno central y un crecimiento constante por parte de los gobiernos locales, lo que se encuentra fundamentado por la implementación de la Estrategia Nacional de Seguridad Ciudadana 2013 - 2018, el cual pone énfasis en el trabajo de patrullaje coordinado entre el serenazgo municipal y el personal policial de la jurisdicción. 
Es preciso resaltar que la participación de los gobiernos regionales en materia de seguridad ciudadana es mínima o marginal y que está destinado principalmente en trasferencias realizadas a los gobiernos locales o inversión en infraestructura a favor de la seguridad ciudadana; pero no en un involucramiento directo en brindar servicios de seguridad ciudadana; lo que sustenta el bajo nivel de asignación y ejecución de presupuesto que en promedio es del $0.25 \%$ del total del presupuesto.

Principales municipalidades de lima metropolitana y su nivel de ejecución de gastos a favor de la seguridad ciudadana.

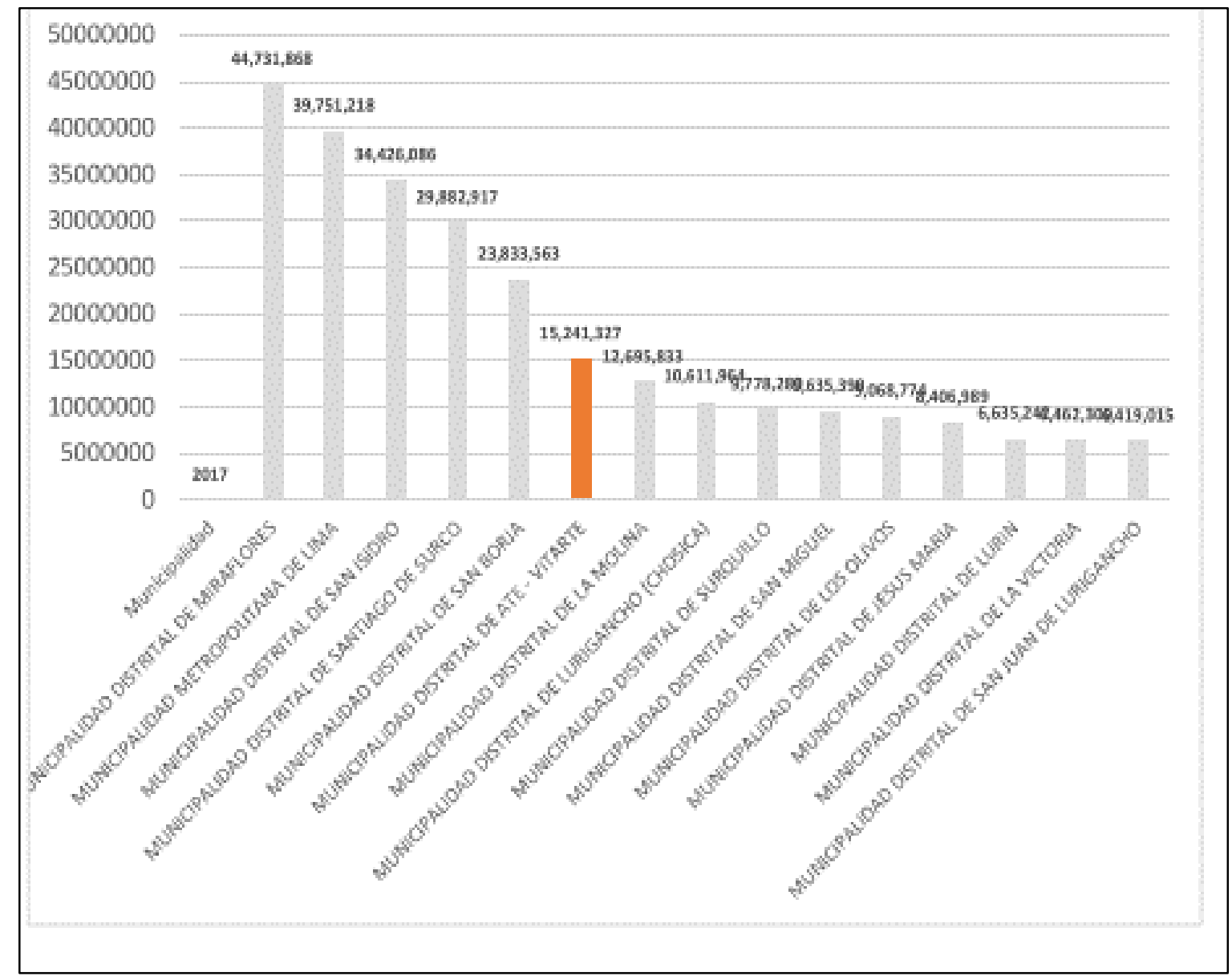

Fuente: INEI 2017

Del Cuadro se puede identificar que las municipalidades distritales que se encuentran ubicadas en zonas de mayor nivel adquisitivo como son Miraflores, San Isidro, Santiago de Surco, tiene presupuestos mayores que los distritos de menor nivel adquisitivo o que cuentan con mayores bolsones de pobreza; es el caso del distrito de Ate, asimismo, es llamativo que el distrito de mayor cantidad de Población de Lima - San Juan de 
Lurigancho, tiene menos presupuesto para seguridad ciudadana que el distrito de Ate que tiene la décima parte de la población o cinco veces menos presupuesto que el distrito de Miraflores que tiene diez veces menos población, lo que demuestra que ante un problema trasversal a todos los distritos como es el caso de la inseguridad ciudadana, cada jurisdicción lo enfrenta de acuerdo a sus posibilidades presupuestales y no en iguales condiciones.

\begin{tabular}{|c|c|c|c|}
\hline \multirow[t]{2}{*}{ ACTIVIDAD DELICTIVA } & \multicolumn{3}{|c|}{ Años } \\
\hline & 2015 & 2016 & 2017 \\
\hline Homicidio calificado & 6 & 0 & 1 \\
\hline Homicidio & 5 & 9 & 4 \\
\hline Feminicidio & 0 & 0 & 1 \\
\hline Sicariato & 0 & 0 & 0 \\
\hline Extorsión & 6 & 11 & 1 \\
\hline Robo simple y agravado & 1,432 & 1,320 & 858 \\
\hline Hurto simple y agravado & 1,800 & 1,927 & 1,826 \\
\hline Tenencia ilegal de armas & 0 & 0 & 21 \\
\hline TID (microcomercialización, posesión y consumo) & 25 & 53 & 53 \\
\hline Estafa & 64 & 60 & 48 \\
\hline Apropiación ilicita & 33 & 42 & 26 \\
\hline Usurpación & 13 & 20 & 18 \\
\hline Delitos contra la libertad sexual & 250 & 192 & 228 \\
\hline Delitos contra la libertad sexual (Secuestros) & 11 & 2 & 3 \\
\hline Delitos contra la fe pública & 15 & 5 & 4 \\
\hline Falsificación de moneda & 0 & 0 & 0 \\
\hline Faltas contra la persona & 1,405 & 618 & 707 \\
\hline Faltas contra el patrimonio & 98 & 210 & 218 \\
\hline Violencia familiar & 948 & 1,667 & 2,824 \\
\hline Abandono de hogar & 1,062 & 1,182 & 1,666 \\
\hline Pandillaje pernicioso & 0 & 0 & 0 \\
\hline Lesiones & 356 & 298 & 333 \\
\hline Exposición y abandono a personas en peligro & 3 & 0 & 1 \\
\hline
\end{tabular}

Según la Estadística policial, se puede apreciar que pese a que los presupuestos destinados a seguridad ciudadana, se han incrementado sostenidamente, no así sucede con los resultados los cuales tiene comportamientos mixtos, al existir algunos tiene un mínimo de reducción como es el caso de los delitos contra el patrimonio (Robo y Hurto), delitos 
contra la libertad sexual y en otros se ha incrementado como el Tráfico Ilícito de Drogas, faltas contra la persona, violencia familiar y lesiones, lo que demuestra que el Presupuesto por resultado no está cumpliendo las metas esperadas.

\begin{tabular}{|c|c|c|c|c|c|c|c|c|}
\hline \multirow[b]{2}{*}{ Ano } & \multirow[b]{2}{*}{ PIA } & \multirow[b]{2}{*}{ PIM } & \multirow[b]{2}{*}{ Certificación } & \multirow[b]{2}{*}{ Ejocución } & \multirow[b]{2}{*}{$\begin{array}{c}\mathrm{Yd \theta} \\
\text { Incremento } \\
\text { Ejec. }\end{array}$} & \multirow[b]{2}{*}{ 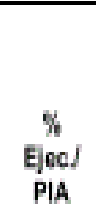 } & \multirow[b]{2}{*}{$\begin{array}{c}\text { s. } \\
\text { Ejec.l } \\
\text { PIM }\end{array}$} & \multirow[b]{2}{*}{$\begin{array}{c}\% \text { EjecJ } \\
\text { Certificasion }\end{array}$} \\
\hline & & & & & & & & \\
\hline 2012 & $2,272,756,572$ & $2,896,214,073$ & $2,799,595,967$ & $2,797,598,446$ & & 101.2 & 96.6 & 99.9 \\
\hline 2013 & $2,546,609,758$ & $3,502,067,952$ & $3,370,467,386$ & $3,349,693,899$ & 16.5 & 101.3 & 95.6 & 99.4 \\
\hline 2014 & $3,678,162,102$ & $4,707,125,481$ & $4,544,663,533$ & $4,509,815,752$ & 25.7 & 101.2 & 95.8 & 99.2 \\
\hline 2015 & $4,209,262,186$ & $5,069,299,007$ & $4,806,219,981$ & $4,754,743,414$ & 5.2 & 101.1 & 93.8 & 98.9 \\
\hline 2016 & $4,256,864,100$ & $4,809,370,229$ & $4,568,276,334$ & $4,463,250,762$ & -6.5 & 101.0 & 928 & 97.7 \\
\hline 2017 & $4,757,051,111$ & $5,311,553,129$ & $5,013,934,555$ & $4,797,129,021$ & 6.9 & 101.0 & 90.3 & 95.7 \\
\hline 2018 & $5,168,549,747$ & $5,739,209,179$ & $5,428,061,978$ & $4,413,936,038$ & -8.7 & 100.9 & 76.9 & 81.3 \\
\hline TOTAL. & $26,889,255,576$ & $32,033,859,050$ & $30,531,219,734$ & $29,084,857,332$ & & 101.1 & 90.8 & 95.3 \\
\hline
\end{tabular}

Fuente: SIAF Amigable, 2018 - Ministerio de Economia y Finanzas.

Respecto al nivel de ejecución de gastos del Programa Presupuestal 030: Reducción de delitos y faltas que afectan la seguridad ciudadana en el periodo $2012-2018$, se desprende que el nivel de ejecución presupuestal, mantiene un ratio de reducción de la ejecución desde un nivel de $96.6 \%$ en el año 2012 hasta un 76.9\% en el año 2018, teniendo un nivel de ejecución promedio de 90.8\%, es decir se devuelve al tesoro público cerca del $10 \%$ del presupuesto anual por falta de ejecución; lo que demuestra que existe aún una limitación en la capacidad de gasto de algunas entidades, principalmente en el rubro inversión. 


\begin{tabular}{|c|c|c|c|c|c|c|}
\hline $\begin{array}{l}\text { PRODUCTOS I } \\
\text { PROYECTOS }\end{array}$ & PIA & PIM & EJECUCION & $\begin{array}{l}\% d e \\
\text { GG/GT } \\
\text { OTAL }\end{array}$ & $\begin{array}{c}\% \text { de } \\
\text { EJECI } \\
\text { PIA }\end{array}$ & $\begin{array}{c}\% \text { de } \\
\text { EJECI } \\
\text { PIM }\end{array}$ \\
\hline $\begin{array}{l}\text { GASTOS DE INVERSION } \\
\text { (PROYECTOS) }\end{array}$ & $210,296,067$ & $212,683,339$ & $123,876,372$ & 3.1 & 58.9 & 58.2 \\
\hline $\begin{array}{l}\text { 3000001, ACCIONES } \\
\text { COMUNES }\end{array}$ & $63,502,548$ & $72,519,802$ & $62,005,218$ & 1.6 & 97.6 & 85.5 \\
\hline $\begin{array}{l}\text { 3000355. PATRULLLAJE } \\
\text { POR SECTOR }\end{array}$ & $1,782,482,209$ & $1,733,992,902$ & $1,724,257,732$ & 43.7 & 96.7 & 99.4 \\
\hline $\begin{array}{l}\text { 3000356. COMUNIDAD } \\
\text { ORGANIZADA A FAVOR } \\
\text { DE LA SEGURIDAD } \\
\text { CIUDADANA }\end{array}$ & $33,065,903$ & $35,829,321$ & $34,493,716$ & 0.9 & 104.3 & 96.3 \\
\hline $\begin{array}{l}\text { 30000422, OPERACIONES } \\
\text { POLICIALES PARA } \\
\text { REDUCIR LOS DELITOS } \\
\text { YFALTAS }\end{array}$ & $1,914,907,566$ & $1,912,393,239$ & $1,907,018,810$ & 48.3 & 99.6 & 99.7 \\
\hline $\begin{array}{l}\text { 3000520, COMISARIAS } \\
\text { CON LAS CONDICIONES } \\
\text { BASICAS PARA EL } \\
\text { SERVICIO A LA } \\
\text { COMUNIDAD }\end{array}$ & $100,883,185$ & $165,854,976$ & $81,868,694$ & 2.1 & 81.2 & 49.4 \\
\hline $\begin{array}{l}\text { 3000781. UNIDADES } \\
\text { ESPECIALIZADAS CON } \\
\text { LAS CONDICIONES } \\
\text { BASICAS PARA } \\
\text { OPERACIONES } \\
\text { POLICIALES }\end{array}$ & $14,418,375$ & $16,765,031$ & $11,836,929$ & 0.3 & 82.1 & 70.6 \\
\hline TOTAL & $4,119,555,853$ & $4,150,038,610$ & $3,945,357,470$ & 100.0 & 95.8 & 95.1 \\
\hline
\end{tabular}

Del presente cuadro se desprende que la mayor cantidad de recursos destinados a la seguridad ciudadana del Programa Presupuestal 030: Reducción de los delitos y faltas que afectan la Seguridad Ciudadana, se destina a la prevención a través del patrullaje y operaciones policiales para reducir los delitos y faltas que ambos suman el 92\% de todo el presupuesto, mientras que el gasto destinado a inversión significa un $8 \%$ del total asignado y ejecutado. 


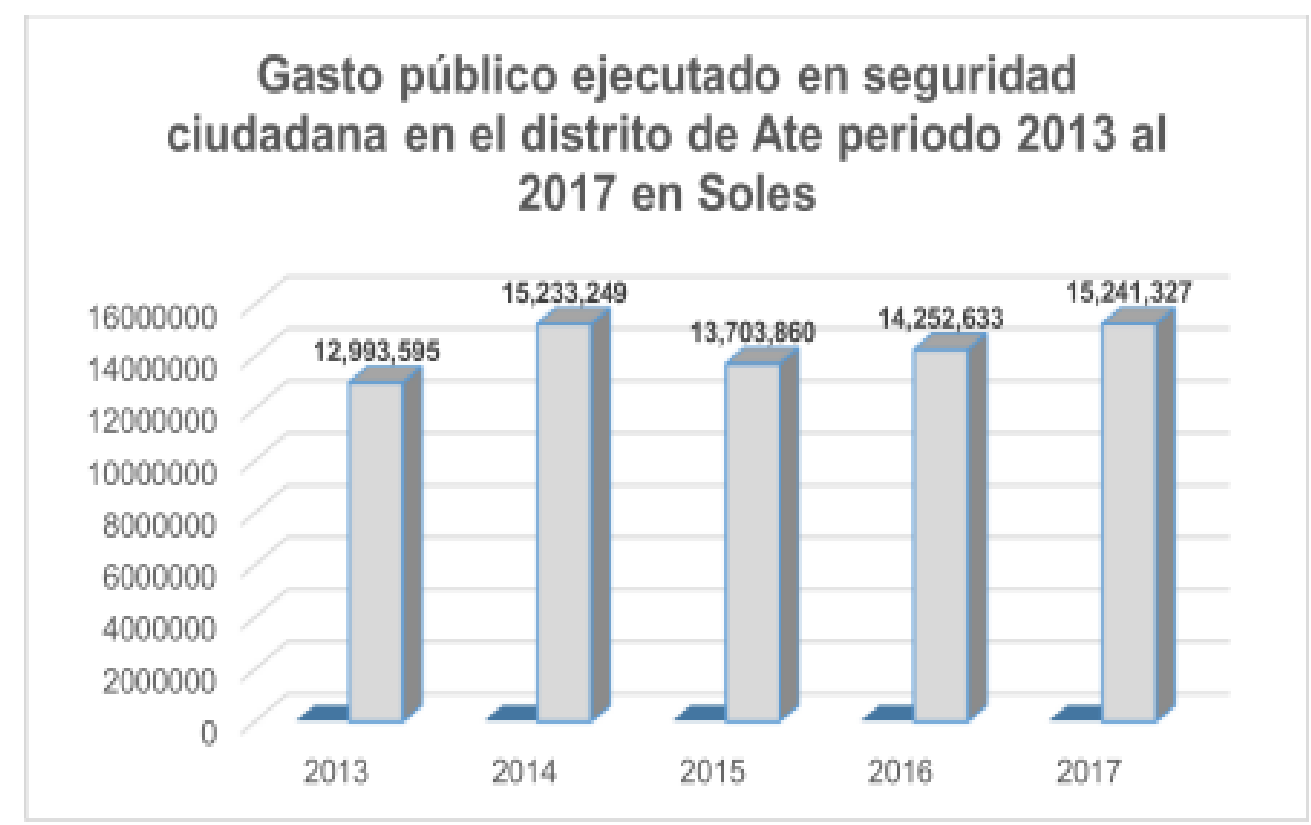

Fuente: Municipalidad distrital de Ate 2018.

Respecto al gasto en materia de seguridad ciudadana aplicado al Distrito de Ate se mantiene en el orden de S/ 15241327.00 Soles entre el periodo comprendido entre los años 2013 al 2017, de un mínimo de S/ 12993595.00 Soles, lo que es la mitad del presupuesto asignado en el distrito de Miraflores.

\begin{tabular}{|c|c|c|c|c|c|c|c|}
\hline Distritos & $\begin{array}{l}\text { Ejecución de } \\
\text { gasto público } \\
2017\end{array}$ & Población & $\begin{array}{c}\text { Tasa de } \\
\text { pobreza } \\
\text { (incidencia) }\end{array}$ & $\begin{array}{l}\text { Superficie } \\
\text { total Km2 }\end{array}$ & $\begin{array}{l}\text { Habitantes } \\
\text { por Km2 }\end{array}$ & $\begin{array}{l}\text { Gasto } \\
\text { percápital } \\
\text { población }\end{array}$ & $\begin{array}{l}\text { Gasto } \\
\text { percápital } \\
\text { superficie }\end{array}$ \\
\hline $\begin{array}{l}\text { MUNICIPALIDAD DISTRITAL } \\
\text { ATE }\end{array}$ & $15,241,327$ & 630,086 & 15.6 & 77.7 & 8,107 & 24 & $196,105.6$ \\
\hline $\begin{array}{l}\text { 150113-301262: } \\
\text { MUNICIPALIDAD DISTRITAL } \\
\text { DE JESUS MARIA }\end{array}$ & $8,406,989$ & 71,589 & 1.2 & 4.6 & 15,665 & 117 & $\begin{array}{r}1,839,603 \\
7\end{array}$ \\
\hline $\begin{array}{l}\text { 150130-301279: } \\
\text { MUNICIPALIDAD DISTRITAL } \\
\text { DE SAN BORJA }\end{array}$ & $23,833,563$ & 111,928 & 0.6 & 10.0 & 11,237 & 213 & $\begin{array}{r}2,392,928 . \\
0\end{array}$ \\
\hline $\begin{array}{l}\text { 150122-301271: } \\
\text { MUNICIPALIDAD DISTRITAL } \\
\text { DE MIRAFLORES }\end{array}$ & $44,731,868$ & 81,932 & 0.2 & 9.6 & 8,516 & 546 & $\begin{array}{r}4,649,882 . \\
3\end{array}$ \\
\hline $\begin{array}{l}\text { 150131-301280: } \\
\text { MUNICIPALIDAD DISTRITAL } \\
\text { DE SAN ISIDRO }\end{array}$ & $34,426,086$ & 54,206 & 0.2 & 11.1 & 4,883 & 635 & $\begin{array}{r}3,101,449 \text {. } \\
2\end{array}$ \\
\hline
\end{tabular}


Respecto al gasto per cápita; es decir, el presupuesto que se destina en seguridad ciudadana por cada habitante se aprecia que en el distrito de Ate se destina un promedio de 24 soles por habitante al año, mientras que en el distrito de San Isidro se destinan 635, Miraflores se destinan 546 soles por habitante al año, lo que demuestra la total asimetría en cuestión de presupuesto para enfrentar el mismo problema, lo que se materializa en menor cantidad de personal, meno $\mathrm{r}$ unidad de unidades móviles, menos horas de patrullaje en comparación con otros distritos; lo que teniendo en cuento el análisis de que a mayor inversión mayores niveles de seguridad, en estos situación presupuestal queda demostrador, es por ello los mayores niveles de inseguridad ciudadana en los distritos donde se destinan menores recursos a la seguridad ciudadana como es el caso del distrito de Ate.

\section{CONCLUSIÓN}

Al realizar el presente estudio, se formuló un análisis de caso y análisis documental del presupuesto asignado a seguridad ciudadano contenido en el Programa Presupuestal 030: Reducción de los delitos y faltas que afectan a la seguridad ciudadana de acuerdo a los alcances y diseño de la investigación, utilizando información de gran valor y confianza como es el SIAF MEF, INEI y la Municipalidad Distrital de Ate, teniendo como usuarios de la seguridad ciudadana a la población del distrito de Ate en el periodo comprendido entre el 2012 al 2018,

De acuerdo con la evaluación de la ejecución presupuestal de la Municipalidad distrital de Ate, se advierte que pese a que ha existido un incremento sostenido en la ejecución de recursos públicos a favor de la seguridad ciudadana en el programa presupuestal 030: Reducción de los delitos y faltas que afectan la seguridad ciudadana, esta no se ha reflejado en la reducción de los niveles de inseguridad teniendo resultados mixtos y en algunos casos se ha incrementado.

La disponibilidad presupuestal que tiene cada entidad municipal de acuerdo a su recaudación de los arbitrios serenazgo o seguridad ciudadana y las trasferencias que realizan los gobiernos regionales o gobierno central el cual es muy asimétrico identificándose asignaciones presupuestales por habitante y por año en el distrito de San Isidro 20 veces mayor que en el distrito de Ate; sin tener en consideración la extensión territorial, densidad poblacional o índice de criminalidad, para enfrentar un mismo problema en la capital de la república, lo que demuestra que existan mayor índice criminal 
en distritos de escasos recursos, pues no cuenta con servicios de seguridad ciudadana eficientes.

\section{LISTA DE REFERENCIAS}

Acad, Programa, Mico D. E. Maestr, and E. N. Gesti. 2021. "Escuela de Posgrado.” 0-2. Anderson, V. (2017). Criteria for evaluating qualitative research. [Criterios de evaluación de la investigación cualitativa]. Human Resource Development Quarterly, 1-9. https://doi.org/10.1002/hrdq.21282

Becerra, J. C. B. (2018). Seguridad ciudadana en Bogotá: Análisis del presupuesto y la eficiencia en el gasto local 2011-2016. Rev. Digital de Derecho Admin., 20, 201.

Casasempere-Satorres, A., \& Vercher-Ferrándiz, M. L. (2020). Análisis documental bibliográfico. Obteniendo el máximo rendimiento a la revisión de la literatura en investigaciones cualitativas. New Trends in Qualitative Research, 4, 247-257.

Chinchilla, L., \& Vorndran, D. (2018). Seguridad ciudadana en América Latina y el Caribe. Desafíos e innovación en gestión y políticas públicas en los últimos, 10.

Contraloría General de la República. 2021. "Normas de Control."

Delgadillo Mendoza, H. (2019). El gasto público en la seguridad ciudadana, Ate 2018.

Enrique, A. M., \& Barrio Fraile, E. (2018). Guía para implementar el método de estudio de caso en proyectos de investigación. Propuestas de investigación en áreas de vanguardia, 159-168.

Hermán William, H. S. (2017). Presupuesto por resultados y la capacitación del personal en la gestión financiera de la Unidad Ejecutora 001: OGA MININTER, Lima, 2016.

Hernández-Sampieri, R., y Mendoza, C. (2018), Metodología de la Investigación: Las rutas Cuantitativa, Cualitativa y Mixta. $1^{\mathrm{a}}$ ed. Ciudad de México: McGraw Hill Interamericana Editores, 2018.

Iazzetta, M. (2019). Seguridad ciudadana y actividad policial comunitaria en Argentina. Estudio de caso en Rosario. Revista Científica General José María Córdova, 17(25), 92-110. https://doi.org/10.21830/19006586.387.

Javier Alva, M. B. (2018). Factores de una política integral del estado para fortalecer la seguridad ciudadana y reducir la delincuencia común. Caso Lima Metropolitana, 2015-2017. 
Ministerio de interior (2021) Programa presupuestal 0030 "Reducción de los delitos y faltas que afectan la seguridad ciudadana. https://www.mef.gob.pe/contenidos/presu_publ/migl/metas/meta04_11_MININ TER_present.pdf.

Moreno, E. J. H. (2019). La planificación y gestión presupuestaria de la unidad ejecutora 005 III Dirtepol Trujillo y su influencia en la optimización de los servicios policiales (Doctoral dissertation, Pontificia Universidad Catolica del PeruCENTRUM Catolica (Peru)).

Ramírez, J. E. P., \& Andia, M. R. C. (2019). Seguridad ciudadana: objetiva, y subjetiva en Mollendo-Arequipa, 2014-2018. REVISTA GOBIERNO Y GESTIÓN PÚBLICA, 6(1), 83-107. DOI: https://doi.org/10.24265/iggp.2019.v6n1.05.

Ramos Arias, O. O. (2019). Políticas públicas de seguridad ciudadana en San Miguel: Estudio de caso del Plan "Vecindario Seguro" en el año 2016.

Rodríguez Valles, O. A. (2017). Evaluación de la ejecución presupuestal de la Policía Nacional del Perú y su implicancia en la Política Pública de Seguridad Ciudadana. Salazar, L. (2020) Investigación Cualitativa: Una respuesta a las Investigaciones Sociales Educativas. CIENCIAMATRIA, $6(11)$ 101-110. https://doi.org/10.35381/cm.v6i11.327

Sánchez Mendoza, V. R. (2017). Presupuesto por resultados y ejecución presupuestal en el Programa Presupuestal 0030 en la Unidad Ejecutora 09 Región Policial Huancayo, Lima, 2017.

Stenfors, T., Kajamaa, A., y Bennett, D. (2020). How to... assess the quality of qualitative research. The clinical teacher, [Cómo... evaluar la calidad de la investigación cualitativa. El profesor clínico]. 17(6), 596-599. https://doi.org/10.1111/tct.13242 